\title{
In situ observation of heat-induced degradation of perovskite solar cells
}

\author{
G. Divitini*1, S. Cacovich ${ }^{1}$, F. Matteocci ${ }^{2}$, L. Cinà ${ }^{2}$, A. Di Carlo², C. Ducati ${ }^{1}$ \\ ${ }^{1}$ Department of Materials Science \& Metallurgy, University of Cambridge, 27 Charles Babbage road, \\ CB3 OFS Cambridge (UK)
}

${ }^{2}$ C.H.O.S.E. (Centre for Hybrid and Organic Solar Energy), Department of Electronic Engineering, University of Rome "Tor Vergata", via del Politecnico 1, Rome 00133, Italy

* Corresponding email: gd322@cam.ac.uk

\begin{abstract}
The lack of thermal stability of perovskite solar cells is hindering the progress of this technology towards adoption in the consumer market. Different pathways of thermal degradation are activated at different temperatures in these complex nanostructured hybrid composites. Thus, it is essential to explore the thermal response of the mesosuperstructrured composite device in order to engineer materials and operating protocols. Here we produce devices according to four well established recipes, and characterise their photovoltaic performance as they are heated within the operational range. The devices are analysed using transmission electron microscopy as they are further heated in situ, to monitor changes in morphology and chemical composition. We identify mechanisms for structural and chemical changes, such as iodine and lead migration, which appear to be correlated to the synthesis conditions. In particular, we identify a correlation between exposure of the perovskite layer to air during processing, and elemental diffusion during thermal treatment.
\end{abstract}

\section{Introduction}

Solar cells based on lead halide perovskite composites have become increasingly popular in the last few years due to a combination of low synthesis cost and high power conversion efficiency, with certified values in excess of $20 \%^{1-5}$. The stability of such devices is however a concern - it is well known that heating at or above around $85^{\circ} \mathrm{C}$, a temperature close to those reached during normal operation in full sunlight, performance degrades rapidly, and such instability is exacerbated by exposure to moisture ${ }^{6-8}$; systematic thermal and ageing studies are required to understand such degradation process ${ }^{9}$. Changes happen in both the organic and inorganic components of the cells; the resilience of the perovskite layer, in particular, is expected to become a limiting factor once different hole conducting materials (or hole-conductor-free cells) are developed ${ }^{10}$. In order to overcome this limitation, it is vital to understand the degradation pathways of the structures involved, which here are observed at nanometer-scale spatial resolution in situ, inside an analytical scanning transmission electron microscope (STEM), while the composition is monitored with elemental mapping through energy-dispersive X-ray analysis (EDX). The analysis of such devices is challenging due to several factors. The spatial dimensions relevant to the fabrication and the operation of the cells are in the $1-100 \mathrm{~nm}$ range, and the materials are easily damaged by exposure to an electron beam in a TEM, requiring to carefully tune the electron dose. The system also includes organic and inorganic components in an assembly with complex chemistry and morphology. Finally, the rapid changes to the devices in air and the low degradation temperatures pose an additional challenge to the experiment, which needs to be timed appropriately and carefully executed. 
The monitoring of this process is made possible by combining several recent advances in TEM technology. The use of high-brightness electron guns and detectors with large collection areas allows the fast acquisition of high quality EDX maps with limited electron dose on the sample ${ }^{11}$; the signalto-noise ratio of the maps can be further increased by applying denoising algorithms (PCA, Principal Components Analysis) within an open-source software suite ${ }^{12-13}$. The development of novel in situ heating holders for TEM, based on micro-heaters and featuring high-stability and fast-response, was also crucial - in particular, the holder used here allows very precise control (sub-degree) at values just above room temperature, as well as providing fast heating and cooling (a few seconds for the temperatures in use in this paper). The good spatial stability of the holder is crucial in acquiring EDX maps.

Four samples, prepared with different processing routes, have been examined under TEM in order to correlate synthesis process, morphology, photovoltaic properties and behaviour under heating. For three samples a double-step fabrication ${ }^{14}$ was employed - here labelled vacuum conversion (sample $A)$, glovebox (B) and air conversion (C, with relative humidity $\sim 50 \%$ ) - while one was manufactured with a single-step approach ( $D$, in glovebox). Specifically, sample $B$ is a representative of established synthesis inside a glove-box, and sample $C$ is interesting due to the processing in humid air ${ }^{15}$. Each synthesis process was optimised separately, and full details are reported in the methods section.

Cross-sectional specimens were extracted as lamellae for TEM analysis using focused ion beam (FIB) milling. The procedure takes a few hours and is carried out in vacuum, hence minimal changes are expected to happen in the samples. Devices were unsealed just before FIB processing, limiting exposure to air to a few minutes. TEM characterisation and in situ heating were performed just after the FIB step, ensuring that specimen were exposed to air for a maximum of 5 minutes before being transferred into vacuum again.

A representative STEM high-angle annular dark field (HAADF, in this case taken from sample B) cross-sectional view of a device is shown in figure 1. The cell is manufactured on FTO-coated glass (Fluorine-doped Tin Oxide), further coated with a compact $\mathrm{TiO}_{2}$ hole blocking layer. A mesoporous $\mathrm{TiO}_{2}$ layer is infiltrated and capped with methyl-ammonium lead iodide (MAPbl 3 ). A spiro-OMeTAD $\left(2,2^{\prime}, 7,7^{\prime}\right.$-Tetrakis-(N,N-di-4-methoxyphenylamino)-9,9'-spirobifluorene) hole transporting layer and a gold electrode complete the cell; platinum is deposited on top during FIB sample preparation as a standard procedure in order to protect the sample during milling. EDX elemental maps are shown in figure 1, showing well defined layers and good infiltration of lead and iodine inside the titania scaffold.

The samples present different morphologies. The images reported in figure 2 are Z-contrast images, where the brightness of a given pixel is proportional to the average atomic number and thickness (in this case constant, around $150 \mathrm{~nm}$, for all samples, in the region of interest). The brightest areas are the Au electrodes, while the organic hole transporter appears black. The titania scaffold appears as a dark feature overlapping with the brighter perovskite layer; darker areas in this region (voids) result from incomplete infiltration of the perovskite inside the scaffold. Samples B and C present good perovskite infiltration inside the titania, as well as a homogeneous perovskite capping layer (around 120 and $200 \mathrm{~nm}$ thick respectively). Sample A has a thin capping layer, and incomplete infiltration in the scaffold can be observed as dark regions near the $\mathrm{TiO}_{2}$ compact film. Sample $\mathrm{D}$, grown with a single step deposition, presents a very inhomogeneous perovskite distribution, leading to micronsized gaps and an infiltration of hole transporter material (HTM) inside the titania scaffold, as well as the formation of voids between the HTM and the Au electrode

The perovskite layers grow differently according to the synthesis conditions - samples A and B present fairly homogeneous layers; sample $C$ has larger, faceted crystals. The perovskite region in sample $\mathrm{C}$ also appears to be composed of two separate layers: the area directly above the titania is 
brighter, suggesting a higher average atomic number, whereas the top layer is darker and more clearly faceted, probably due to a higher degree of crystallinity.

In all samples, voids are visible in the spiro-OMeTAD layer across the entire depth (see Supplementary Figure 1). With a size up to $50 \mathrm{~nm}$, similar features have been previously observed by AFM (Atomic Force Microscopy, seen in top view) and SEM (Scanning Electron Microscopy, in crosssection) and are suspected to play a major role in the infiltration of moisture inside the cell ${ }^{16}$. These voids are introduced during fabrication and are not influenced by the thermal treatments.

\section{Photovoltaic characterisation}

A characterisation of the photovoltaic properties was carried out at room temperature and upon heating. Twin samples (4 per type), nominally identical to the ones used for TEM analysis, were heated ex-situ up to $90^{\circ} \mathrm{C}$. This is representative of the temperatures the cell would operate at under full illumination; a reversible behaviour of the parameters is observed for heating within this temperature range (sample B shown in Figure 3, see Supplementary Figure 2 for the other samples) - upon cooling, the original performance is recovered in all cases except for sample $D$, where the inhomogeneity of the active layer can lead to connectivity issues. In the comparison between all sample types, reported in Figure 4, samples B and $C$ show relatively stable power conversion efficiency (PCE, change up to $10 \%$ ). Sample A exhibits a strong hysteresis. It is known that mesoporous $\mathrm{TiO}_{2}$ significantly reduces hysteresis compared to planar cells ${ }^{17}$; in the case of sample $A$ we attribute such effect to the lack of a continuous perovskite capping layer, which can result in poor connectivity of the domains infiltrating the mesoporous volume. In all samples an overall decay of the PCE is observed for increasing temperature. This is related to a decrease in $V_{\text {oc }}$ (open-circuit voltage - decrease of about $20 \%$ in all samples), partially compensated, in samples B and C, by an increase in fill factor. The change in $V_{o c}$ is consistent with results in the literature and ascribed to increased recombination ${ }^{18}$. The $V_{o c}$ decrease in sample $C$ is slightly slower than in $B$ or $D$; we hypothesise that this could be caused by the presence of iodine dopants/vacancies at the interfaces (mainly between perovskite and HTM) affecting the interfacial energy mismatch ${ }^{19}$. $\mathrm{J}_{\mathrm{sc}}$ (short-circuit current) is moderately reduced with heating up to around $50^{\circ} \mathrm{C}$ in $\mathrm{B}$ and $\mathrm{C}$; since previous work in the literature showed a constant $J_{s c}$ for hole transporter-free perovskite cells ${ }^{20}$, we attribute the observed reduction to a change in the transport properties of spiro-OMeTAD. At a temperature corresponding to the perovskite phase transition $\left(\sim 60^{\circ} \mathrm{C}\right)$ the short circuit current starts moderately increasing ${ }^{21}$. This effect is stronger in sample $D$, where the film is less constrained due to the presence of voids in the structure. The observed fill factor increases for increasing temperature, due to a decrease in the series resistance (see Supplementary Figures 3 and 4). This is due to the resistivity decrease of both perovskite ${ }^{21}$ and spiro-OMeTAD ${ }^{22}$. As the temperature is brought down, the devices (A, B and $C$ ) recover the initial performance, showing the reversibility of the phase transformation.

Above $90^{\circ} \mathrm{C}$ the $\mathrm{HTM}$, here doped spiro-OMeTAD ${ }^{23}$, is known to degrade non-reversibly due to the evaporation of the dopants (Li-TFSI - lithium bis(trifluoromethanesulfonyl)imide - and TBP - tertbutylpyridine). TBP could evaporate after heating stress at lower temperature $\left(\sim 85^{\circ} \mathrm{C}\right)$ with respect to the TBP boiling point $\left(197^{\circ} \mathrm{C}\right)$, as already shown for solid-state dye solar cells by Bailie et al. ${ }^{24}$

\section{TEM in situ heating}

Cross-sectional views of all samples, at different stages of heating, are shown in figure 2. Videos of the STEM images acquired during the heating ramp for each sample are available as Supplementary Videos 1 (sample A) to 4 (sample D). To enhance speed and reproducibility of in situ heating cycles, a reference temperature of $50^{\circ} \mathrm{C}$ was chosen. Photovoltaic measurements show that devices are stable at this temperature and the initial room temperature performance can be restored after 
cooling. The thermal decomposition was "frozen" after each heating step by bringing the temperature down to $50^{\circ} \mathrm{C}$ during the acquisition of the EDX maps, which took about 20 minutes per map. The heating profile, reported in Supplementary Figure 5, was chosen in order to explore the full degradation process, and includes steps at $100,150,175,200,225,250^{\circ} \mathrm{C}$. Initial steps are prolonged for 30 minutes, while the later ones are shortened to 15 minutes due to the faster sample dynamics.

No change in the perovskite layer is visible in the HAADF images for heating up to $150^{\circ} \mathrm{C}$, even if the light conversion properties of the devices appear to degrade irreversibly above $90^{\circ} \mathrm{C}$. This suggests that the main mechanism for performance degradation at low temperatures is related to the HTM, and in particular to how the HTM and the additives react upon heating. The low concentration and low atomic number of the additives are beyond the detection and mapping capabilities of STEMEDX.

The first noticeable change in the perovskite layer occurs on device $\mathrm{C}$ after heating at $150^{\circ} \mathrm{C}$. Due probably to heat-assisted electron beam damage, holes start forming in the region of the perovskite layer close to the interface with the titania scaffold. The methylammonium lead iodide layer, synthesised in air in this case, presents two different levels of contrast in STEM-HAADF, with a brighter region where combined electron beam / thermal degradation happens earlier than in the darker region. This particular behaviour, not observed in the other devices, can be caused by the warm air flow used during the drying step of the $\mathrm{Pbl}_{2}$ layer that induces the incorporation of a low concentration of oxygen and moisture; elemental analysis of the two perovskite regions with different electron density shows comparable stoichiometry within the EDX accuracy and can not account for the change in HAADF intensity, therefore suggesting a difference in crystallinity.

As the temperature increases, the perovskite layer starts degrading and small particles, which EDX suggests to be $\mathrm{Pbl}_{2}$, form at the interface with the FTO layer and start migrating and coalescing in specimens A, B and D. These features are visible in Figure 2 as bright spots. This is a phenomenon due to the intrinsic 2D nature of the specimen (a lamella about $150 \mathrm{~nm}$ thick), where particles can move on the surface of the cross-section. Lead and iodine originate from the perovskite layer; the preferential formation of $\mathrm{Pbl}_{2}$ aggregates over the FTO layer can be explained by the presence of a rougher surface - the FTO layer, being below a mesoporous layer, presents fine roughness due to FIB milling and the "curtaining" effect ${ }^{25}$. Such high mobility of atomic species on heating of $\mathrm{MAPbl}_{3}$ has recently been observed in samples heated ex situ at $85^{\circ} \mathrm{C}$ for $24 \mathrm{~h}$ while exposed to different gases $^{26}$. The later onset of this phenomenon observed here could be attributed to the surrounding environment (heating here is conducted in vacuum) and to the timescale of the process (the heating steps in this work are 30' each). Interestingly, the aggregation of such particles is not visible in sample $\mathrm{C}$. This is ascribed to the different dynamics of the heating process, leading to elemental diffusion of $\mathrm{Pb}$ and I rather than coalescence.

The temperature ramp is continued on all samples up to $250^{\circ} \mathrm{C}$, where the perovskite component of the cell disappears within a few minutes, while the overall structure is kept in place by the FTO and metal electrodes. For reference, a sample prepared from the same device after 2 months of exposure to air (in the dark) was compared to the different stages of the heating ramp, finding a comparable degradation to the step at $200^{\circ} \mathrm{C}$ (Supplementary Figure 6).

The diffusion of heavy elements inside the spiro-OMeTAD can be seen in the HAADF signal as sample $C$ is heated (Figure 5). EDX mapping identifies the first migrating species to be iodine. This behaviour has been suggested in the literature ${ }^{19-27}$, although it had never been directly observed in real time in a TEM. On the pristine sample the presence of iodine extends for $100 \mathrm{~nm}$ beyond the limit of the perovskite layer under a concentration gradient; the iodine front advances towards the Au electrode under heating at increasing diffusion speed, reaching a depth of 50 to $80 \mathrm{~nm}$ after the last thermal step. Elemental migration of lead in the same direction is also observed, although it is triggered at 
higher temperature (starting from $\sim 175^{\circ} \mathrm{C}$ ). Since partial elemental diffusion is observed for iodine before heating, we suggest that this process might be happening as part of the ageing process, and could be responsible for the long term loss of performance at room temperature for devices fabricated without moisture control, outside a dry-box.

Analysis of the STEM-HAADF images can provide a quantitative estimate of the degradation of the cell structure with time. As the sample thickness is constant and the $\mathrm{TiO}_{2}$ layer doesn't degrade at such temperatures, the signal decays as the inorganic components of the perovskite diffuse or evaporate. Figure 6 reports the profile of the HAADF signal for samples B and C, averaged over the entire film, divided into the infiltrated and the capping regions. As seen in Figure 2, very little changes until $150^{\circ} \mathrm{C}$, after which a steady decay is observed. The loss in HAADF signal upon heating is slower in the mesoporous regions compared to the capping ones, suggesting a higher stability. This resilience towards heating combines with robustness upon light irradiation and oxygen exposure that has been demonstrated in the literature ${ }^{28}$. Surprisingly, the signal decay in sample $C$ is slower than in sample B, even though the former was exposed to air and moisture during the synthesis. A possible explanation is that the incorporation of low concentrations of oxygen and water in the perovskite film, while determining local degradation, also induce pinning sites and prevent the diffusion of elemental $\mathrm{Pb}$ and $\mathrm{Pbl}_{2}$ towards the FTO electrode which was observed in other samples.

\section{Conclusions}

In this work we reported the in situ TEM observation of the thermal response of four perovskitebased solar cells heated to and above operational temperatures until full degradation of the composite device. The fabrication routes strongly affect the perovskite coverage and scaffold infiltration, as well as the degradation mechanisms observed. In all samples the perovskite layer is shown to be stable for short times until $150^{\circ} \mathrm{C}$. Exposure to air during fabrication results in an inhomogeneous perovskite layer, which presents a different degradation pathway compared to devices processed in a glovebox; the device processed in air also shows significant migration of iodine into the hole transporting layer. These results, obtained with imaging and chemical analysis at nanometre scale, provide new insight on the effective morphologies of perovskite-based solar cells and on the correlations between morphology, chemical composition and thermal stability of the meso-superstructured functional composites.

\section{Methods}

Devices details

Common to all the samples

A raster scanning laser ( $\mathrm{Nd}: \mathrm{YVO}_{4}$ pulsed at $30 \mathrm{kHz}$ average output power $\mathrm{P}=10 \mathrm{~W}$ ) was used to etch the FTO/glass substrates (Pilkington, $8 \Omega \mathrm{cm}^{-1}, 25 \mathrm{~mm} \times 25 \mathrm{~mm}$ ). The patterned substrates were cleaned in an ultrasonic bath, using detergent with de-ionized water, acetone and isopropanol (10 minutes for each step). A 80nm-thick patterned blocking $\mathrm{TiO}_{2}$ layer $\left(\mathrm{BL}^{-\mathrm{TiO}_{2}}\right)$ layer was deposited onto the patterned FTO using spray pyrolysis in according with a previously reported procedure ${ }^{29}$. A $250 \mathrm{~nm}$-thick mesoporous $\mathrm{TiO}_{2}$ layer (18NR-T paste, Dyesol) diluted with terpineol and ethylcellulose was screen-printed over the $\mathrm{BL}^{-} \mathrm{TiO}_{2}$ surface and sintered at $480^{\circ} \mathrm{C}$ for $30 \mathrm{~min}$. The final thickness was measured by profilometer (Dektak Veeco 150). Different approaches for the perovskite layer deposition were used for each sample, as discussed below.

The hole-transport layers was deposited by spin-coating a $75 \mathrm{mg} \mathrm{ml}^{-1}$ solution of 2,20,7,70-tetrakis( $\mathrm{N}, \mathrm{N}$-dip-methoxyphenylamine)9,9'-spirobifluorene (spiro-OMeTAD) doped with $8 \mu \mathrm{l}$ of tertbutylpyridine and $12 \mu \mathrm{l}$ of lithium bis(trifluoromethanesulfonyl)imide (Li-TFSI) solution (520 mg in 1 
$\mathrm{ml}$ of acetonitrile). Samples were introduced into a high vacuum chamber $\left(10^{-6} \mathrm{mbar}\right)$ to thermally evaporate Au back contacts (nominal thickness $100 \mathrm{~nm}$ ).

Masked devices were tested under a solar simulator (ABET Sun 2000, class A) at AM1.5G and 100 $\mathrm{mW} \mathrm{cm}{ }^{-2}$ illumination conditions calibrated with a certified reference Si Cell (RERA Solutions RR1002). Incident power was measured with a Skye SKS 1110 sensor. The absorbance was measured with a BLACK-Comet UV-VIS Spectrometer. PV parameters were measured on 8 samples for each fabrication method for the batch being analysed, of which 4 were used for the measurements reported in Figure 4. Extensive PV characterisation has been carried out on a set of 160 cells, as shown in Supplementary Figures 7 to 9. Before TEM measurements, samples were glass-glass encapsulated with a thermoplastic gasket to protect the perovskite layer from moisture. This allows an easy encapsulation removal just before the TEM sample preparation.

Sample A: $\mathrm{Pbl}_{2}$ powder (Aldrich, 99\%) was dissolved in DMF, using a concentration of $400 \mathrm{mg} \mathrm{ml}^{-1}$ and stirred at $70^{\circ} \mathrm{C}$. The hot $\mathrm{Pbl}_{2}$ solution was spin-coated on mesoporous titania scaffold at 4000 RPM for $30 \mathrm{~s}$ on substrates preheated to $70^{\circ} \mathrm{C}$. These were then dried at $120^{\circ} \mathrm{C}$ for $1 \mathrm{~h}$ in air to remove the solvent and drive the crystallization. The perovskite film was grown by dispersing MAI powder on the sample on a hotplate. The samples and the powder were put under low vacuum (0.02 mbar) and heated at $150^{\circ} \mathrm{C}$.

Sample B: The lead iodide solution ( $\mathrm{Pbl}_{2}$ in $\mathrm{N}, \mathrm{N}$-dimethylformamide, $460 \mathrm{mg} \mathrm{ml}^{-1}$ ) was deposited by spin coating at $6000 \mathrm{rpm}$ for $10 \mathrm{sec}$ and then dried at $70^{\circ} \mathrm{C}$ for 1 hour. $\mathrm{CH}_{3} \mathrm{NH}_{3} \mathrm{Pbl}_{3}$ crystallization was achieved by dipping the $\mathrm{Pbl}_{2}$ layers in a methylammonium iodide solution $\left(\mathrm{CH}_{3} \mathrm{NH}_{3} \mathrm{l}\right.$ in anhydrous 2propanol $10 \mathrm{mg} \mathrm{ml}^{-1}$ ) for $10 \mathrm{sec}$ and washing immediately with 2-propanol by spin coating at 6000 rpm for $10 \mathrm{sec}$, followed by drying at $70^{\circ} \mathrm{C}$ for $30 \mathrm{~min}$.

Sample C: The lead iodide solution ( $\mathrm{Pbl}_{2}$ in $\mathrm{N}, \mathrm{N}$-dimethylformamide, $330 \mathrm{mg} \mathrm{ml}^{-1}$ ) layer was deposited by blade coating; the blade was set to a height of $100 \mu \mathrm{m}$ above the sample surface with a speed of $40 \mathrm{~mm} \mathrm{~s}^{-1}$. To obtain a compact and flat layer, an air flow (flow $17 \mathrm{~m}^{3} \mathrm{~s}^{-1}$, temperature $100^{\circ} \mathrm{C}$ ) was used to quickly evaporate the solvent after deposition. $\mathrm{CH}_{3} \mathrm{NH}_{3} \mathrm{Pbl}_{3}$ crystallization was achieved dipping the $\mathrm{Pbl}_{2}$-based samples in a methylammonium iodide solution $\left(\mathrm{CH}_{3} \mathrm{NH}_{3} \mathrm{I}\right.$ in anhydrous isopropanol, $10 \mathrm{mg} \mathrm{ml}^{-1}$, purchased from Dyesol) for 30 minutes.

Sample D: Grown using methylammonium iodide (Dyesol) and $\mathrm{PbCl}_{2}$ (Aldrich, 98\%). Both precursors were used as received. The film was deposited by spin coating (2000 rpm for $60 \mathrm{~s}$ ) from a dimethylformamide (DMF) solution of methylammonium iodide and $\mathrm{PbCl}_{2}$ (3:1 $\mathrm{M}$ ratio) in inert atmosphere, which formed the perovskite after heating to $90^{\circ} \mathrm{C}$ for $60 \mathrm{~min}$.

The initial PCE values for the devices in the batch were $9.6 \pm 1.3$ (A), $12.6 \pm 1.3$ (B), $11.7 \pm 1.6$ (C), 7.9 \pm 1.8 (D).

\section{Microscopy and data analysis}

Samples for TEM were prepared in a FEI Helios Nanolab FIB/SEM as a lamella on a heating chip by DENSsolutions. STEM/EDX was carried out in a FEI Osiris ( $200 \mathrm{kV}$ acceleration voltage) equipped with a X-FEG gun and a Bruker Super-X EDX detector. EDX maps were acquired over an area of around 1 $\mu \mathrm{m}^{2}$ (with small variations due to the thickness of the different samples) with sampling every $10 \mathrm{~nm}$ and a dwell time of $100 \mathrm{~ms}$ per pixel, using a beam current of $700 \mathrm{pA}$. The specimens are not damaged by this irradiation, as can be confirmed by comparison of the different maps acquired up to $150^{\circ} \mathrm{C}$. EDX spectrum images were denoised using the PCA routines implemented in Hyperspy, and the maps were extracted using the same software suite. In situ heating was performed using a Wildfire holder by DENSsolutions. 


\section{References}

1. Green, M., Emery, K., Hishikawa, Y., Warta, W. \& Dunlop, E. D. Solar cell efficiency tables (Version 45). Prog. in Photovolt: Res. Appl. 23, 1-9 (2015)

2. Snaith, H. J. Perovskites: The emergence of a new era for a low-cost, high efficiency solar cells, J. Phys Chem. Lett. 4, 3623-3630 (2013)

3. Grätzel, M. The light and shade of perovskite solar cells. Nature Mater. 13, 838-842 (2014)

4. Green, M. A., Ho-Baillie, A. \& Snaith, H. J. The emergence of perovskite solar cells. Nature Photon. 8, 506-514 (2014).

5. Song, T.-B. et al. Perovskite solar cells: film formation and properties. J. Mater. Chem. A 3, 9032-9050 (2015)

6. Han, Y. et al. Degradation observations of encapsulated planar $\mathrm{CH}_{3} \mathrm{NH}_{3} \mathrm{Pbl}_{3}$ perovskite solar cells at high temperatures and humidity. J. Mater. Chem. A 3, 8139-8147 (2015)

7. Yang, J, Siempelkamp, B. D., Liu, D. \& Kelly, T. L. Investigation of CH3NH3Pbl3 Degradation Rates and Mechanisms in Controlled Humidity Environments Using in Situ Techniques. ACS Nano 9 (2), 1955-1963 (2015)

8. Habisreutinger, S. N. et al. Carbon Nanotube/Polymer Composites as a Highly Stable Hole Collection Layer in Perovskite Solar Cells. Nano Lett. 14, 5561 - 5568 (2014)

9. Stranks, S. D. \& Snaith, H. J. Metal-halide perovskites for photovoltaic and light-emitting devices. Nature Nanotech. 10, 391-402 (2015)

10. Mei, A. et al. A hole-conductor-free, fully printable mesoscopic perovskite solar cell with high stability. Science 345, 295-298 (2014)

11. Terborg, R. \& Rohde, M. Application of High Energy-resolution Silicon Drift Detectors (SDD) for Quantitative Light Element Analysis. Microsc. Microanal. 9, 120-121 (2003)

12. de la Peña, F. et al. Hyperspy: Hyperspectral data analysis toolbox, doi: 10.5281/zenodo.16850 (2015)

13. Burdet, P., Croxall, S. A. \& Midgley, P. A. Enhanced quantification for 3D SEM/EDS: Using the full set of available X-ray lines. Ultramicroscopy 148, 158-167 (2015)

14. Burschka, J. et al. Sequential deposition as a route to high-performance perovskitesensitized solar cells. Nature 499, 316-319 (2013)

15. Ko, H.-S., Lee, J.-W. \& Park, N.-G. $15.76 \%$ efficiency perovskite solar cells prepared under high relative humidity: importance of $\mathrm{Pbl2}$ morphology in two-step deposition of $\mathrm{CH}_{3} \mathrm{NH}_{3} \mathrm{Pbl}_{3}$. J. Mater. Chem. A 3, 8808-8815 (2015)

16. Hawash, Z., Ono, L. K., Raga, S. R., Lee, M. V. \& Qi, Y. Air-Exposure Induced Dopant Redistribution and Energy Level Shifts in Spin-Coated Spiro-MeOTAD Films. Chem. Mater. 27 (2), 562-569 (2015) 
17. Kim, H.-S. \& Park, N.-G. ] Parameters Affecting I-V Hysteresis of $\mathrm{CH}_{3} \mathrm{NH}_{3} \mathrm{Pbl}_{3}$ Perovskite Solar Cells: Effects of Perovskite Crystal Size and Mesoporous $\mathrm{TiO}_{2}$ Layer. J. Phys. Chem. Lett. 5, 2927-2934 (2014)

18. Ono, L. K., Raga, S. R., Wang, S., Kato, Y. \& Qi, Y. Temperature-dependent hysteresis effects in perovskite-based solar cells. J. Mater. Chem. A 3, 9074-9080 (2015)

19. Azpiroz, J. M., Mosconi, E., Bisquert, J. \& De Angelis, F. Defect migration in methylammonium lead iodide and its role in perovskite solar cell operation. Energy Environ. Sci. 8, 2118-2127 (2015)

20. Aharon, S., Dymshits, A., Rotem, A. and Etgar, L. Temperature dependence of hole conductor free formamidinium lead iodide perovskite based solar cells. J. Mater. Chem. A 3, 9171-9178 (2014)

21. Stoumpos, C. C., Malliakas, C. D. \& Kanatzidis, M. G. Semiconducting Tin and Lead lodide Perovskites with Organic Cations: Phase Transitions, High Mobilities, and Near-Infrared Photoluminescent Properties. Inorg. Chem. 52 (15), 9019-9038 (2013)

22. Dualeh, A., Moehl, T., Nazeeruddin, M. K. \& Grätzel, M. Temperature Dependence of Transport Properties of Spiro-MeOTAD as a Hole Transport Material in Solid-State DyeSensitized Solar Cells. ACS Nano 7 (3), 2292-2301 (2013)

23. Nguyen, W. H., Bailie, C. D., Unger, E. L. \& McGehee, M. D. Enhancing the Hole-Conductivity of Spiro-OMeTAD without Oxygen or Lithium Salts by Using Spiro(TFSI $)_{2}$ in Perovskite and Dye-Sensitized Solar Cells. J. Am. Chem. Soc. 136 (31), 10996-11001 (2014)

24. Bailie, C. D., Unger, E. L., Zakeeruddin, S. M., Grätzel, M. \& McGehee, M. D. Melt-infiltration of spiro-OMeTAD and thermal instability of solid-state dye-sensitized solar cells. Phys. Chem. Chem. Phys. 16, 4864-4870 (2014)

25. Munroe, P. R. The application of focused ion beam microscopy in the material sciences. Mater. Charact. 60, 2-13 (2009)

26. Conings, B. et al. Intrinsic Thermal Instability of Methylammonium Lead Trihalide Perovskite. Adv. Energy Mater. 5, 1500477 (2015)

27. Eames, C. et al. Ionic transport in hybrid lead iodide perovskite solar cells. Nature Commun. 6, 7497 (2015)

28. O'Mahony, F. T. F. et al. Improved environmental stability of organic lead trihalide perovskite-based photoactive-layers in the presence of mesoporous $\mathrm{TiO}_{2}$. J. Mater. Chem. A 3, 7219-7223 (2015)

29. Matteocci, F. et al. Blocking layer optimisation of poly(3-hexylthiopene) based Solid State Dye Sensitized Solar Cells. Org. Electron. 14, 1882-1890 (2013) 


\section{Acknowledgements}

G.D., S.C., and C.D. acknowledge funding from ERC under grant number 259619 PHOTO EM. C.D. acknowledges financial support from the EU under grant number 312483 ESTEEM2. F.M., L.C. and A.D.C. acknowledge funding from "Polo Solare Organico" Regione Lazio, the "DSSCX" MIURPRIN2010 and FP7 ITN "Destiny". G.D and S.C. thank Dr. Francisco de la Peña and Dr. Pierre Burdet for assistance with PCA analysis.

\section{Author Contributions}

G.D., F.M., A.D.C and C.D. conceived and designed the experiment. F.M. produced the devices. F.M. and L.C. carried out photovoltaic characterisation. G.D. and S.C. carried out sample preparation, electron microscopy and data analysis. All authors contributed to the discussion of the results and to the writing of the manuscript.

\section{Additional Information}

Supplementary information is available online. Reprints and permissions information is available online at www.nature.com/reprints. Correspondence and requests for materials should be addressed to G.D.

\section{Competing Financial Interests Statement}

The authors declare no competing financial interests.

\section{Figures}
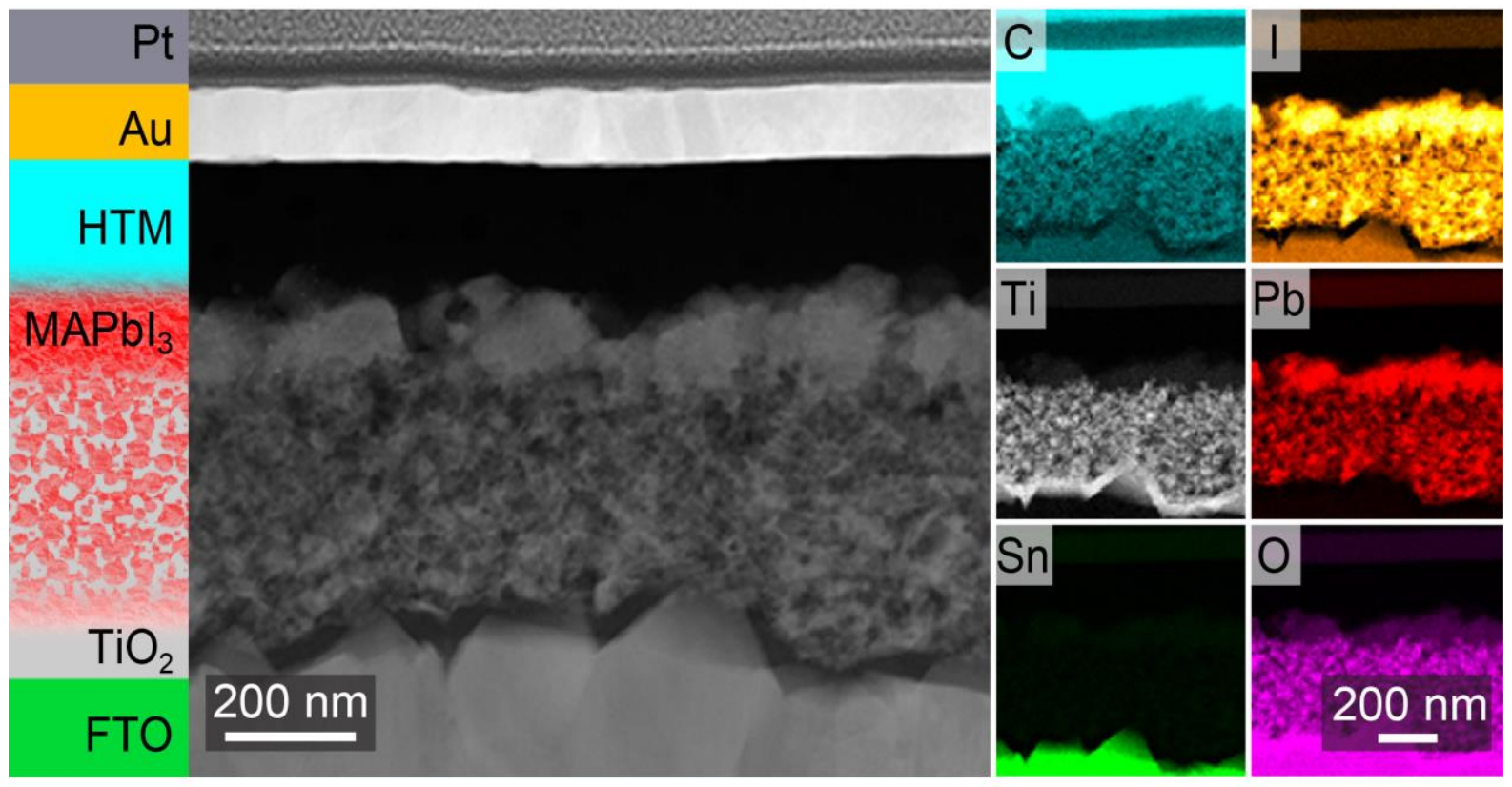

Figure 1 - Cross-sectional view. (Sample B - double step in glovebox) STEM-HAADF image, showing all the layers in the device, labelled on the schematic on the left, and associated EDX elemental maps of the same area (the same scale bar applies to all elemental maps). 


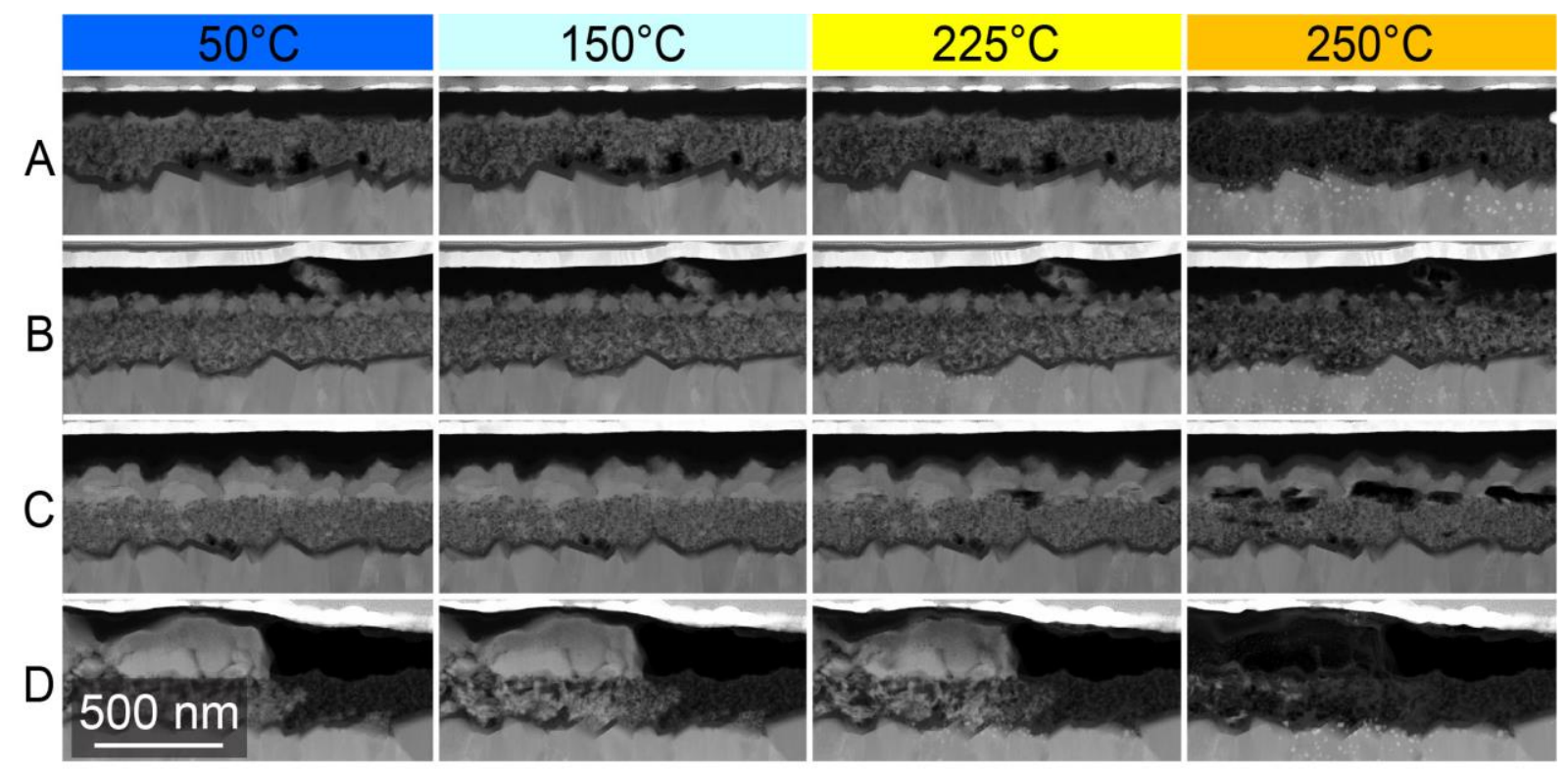

Figure 2 - Cross-sectional views under heating. STEM-HAADF images of all samples at different stages of heating (scale bar applies to all panels), with $50^{\circ} \mathrm{C}$ acting as base temperature for the TEM analysis ( $\mathrm{A}$ - vacuum, B - glovebox, C - air, D - single step). In all samples no visible changes occur in the perovskite layer until $150^{\circ} \mathrm{C}$. In HAADF imaging the brightness is directly proportional to average atomic number and thickness.
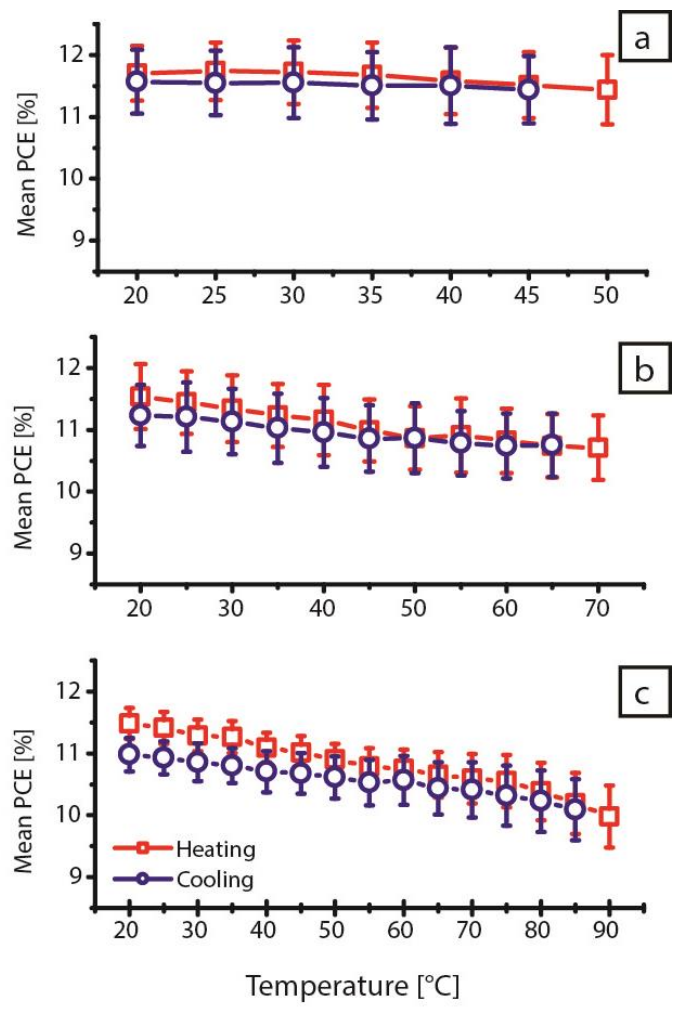

Figure 3 - Thermal cycling. Reversible change in photovoltaic properties of sample B upon heating to $90^{\circ} \mathrm{C}$ and cooling to room temperature in 3 sequential cycles, up to 50 (a), 70 (b) and $90^{\circ} \mathrm{C}$ (c) respectively. Error bars display the standard deviation over the 4 devices. 

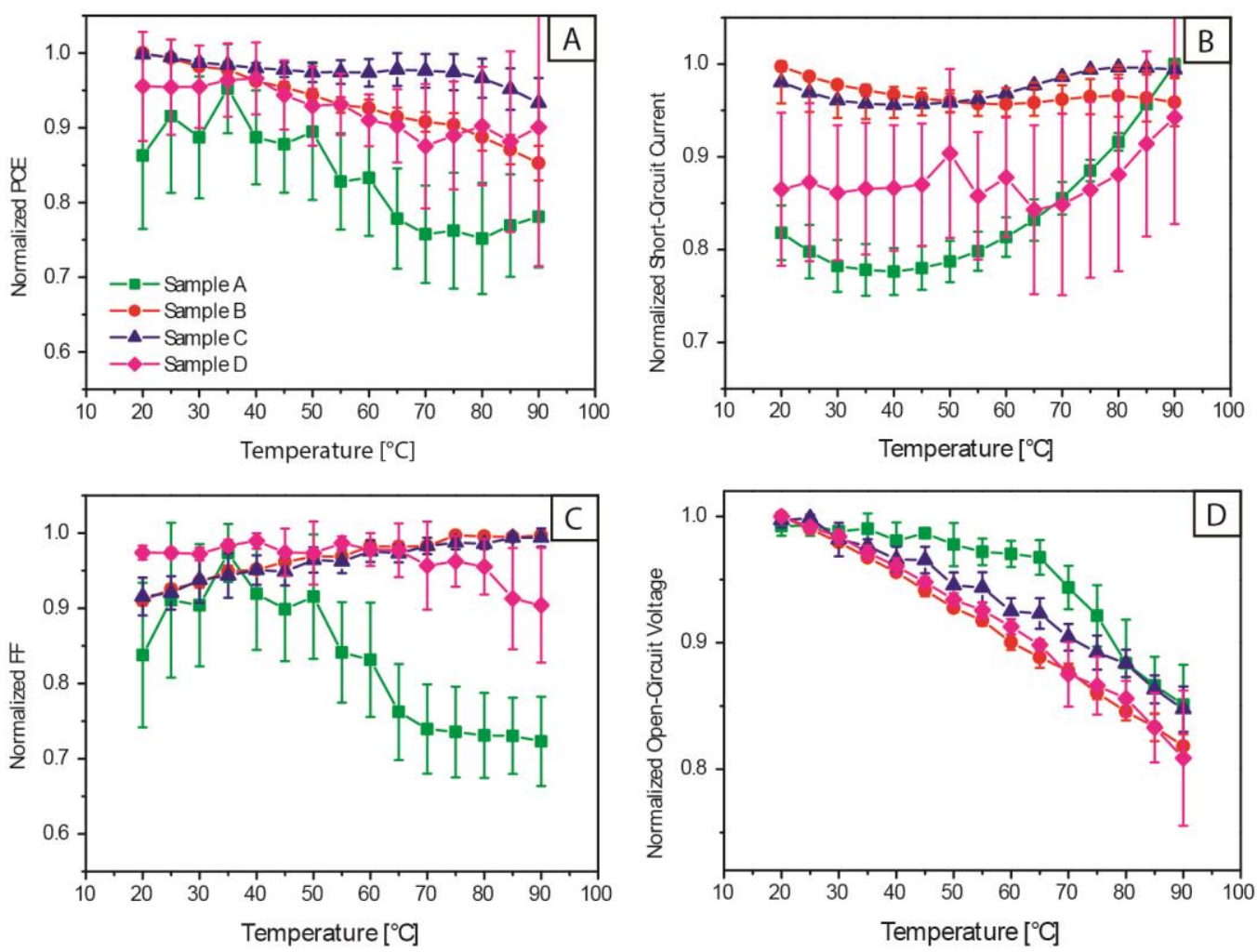

Figure 4 - Photovoltaic properties. Photovoltaic parameters (a) PCE - power conversion efficiency, b) short-circuit current, c) FF - fill factor and d) open-circuit voltage) for all cells. The same colour code has been used for all panels. Error bars indicate the standard deviation, 4 devices per type were used.

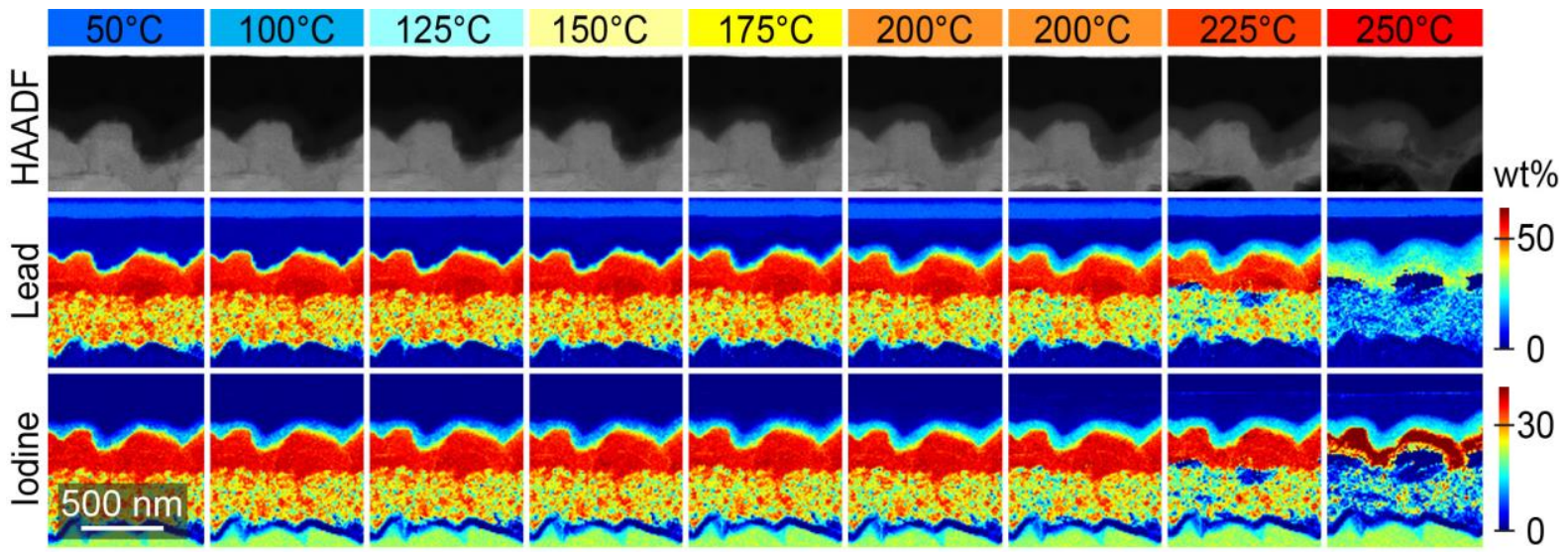

Figure 5 - Temperature evolution of elemental migration. (Sample C) HAADF images and EDX elemental maps for iodine and lead acquired after heating at different temperatures. The heating steps were carried out for $30^{\prime}$ up to $175^{\circ} \mathrm{C}$, and for $15^{\prime}$ from $200^{\circ} \mathrm{C}$ due to the faster sample dynamics. The same scale bar applies to all panels. Diffusion of iodine into the HTM is clearly visible at low temperature, lead migration is triggered at higher temperature $\left(\sim 175^{\circ} \mathrm{C}\right)$. 


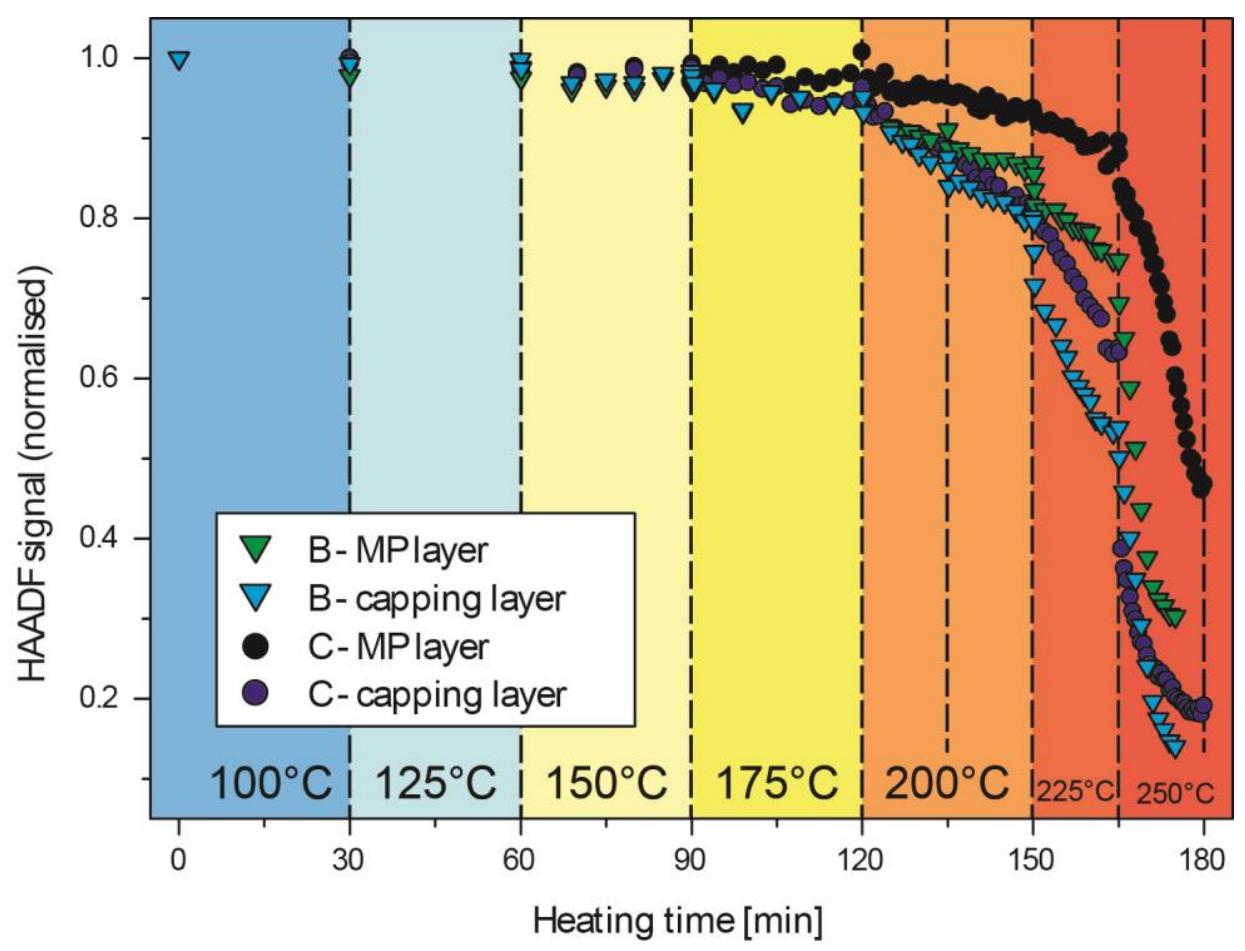

Figure 6 - STEM-HAADF signal evolution. Variation of the STEM-HAADF signal during the heating ramps for samples $B$ and $C$, for both the mesoporous and the capping regions. EDX maps were acquired at $50^{\circ} \mathrm{C}$ at every vertical line in the graph. 